\title{
Variations of Hospital Costs of Spinal Fusion Procedures in Different Regions and Payer Types
}

\section{Ravi Chinta*}

Department of Management, Huizenga College of Business and Entrepreneurship, USA

\begin{abstract}
Back pain continues to be a leading cause of disability in the United States and is one of the most common reasons for seeking consultation with a physician Nonsurgical interventions remain the first-line of therapy; however, many patients eventually progress to a level of severity that requires surgical treatments such as spinal fusion. Spinal fusion has accounted for the highest total cost among the surgical procedures. The Medicare program reported reimbursing US hospitals $\$ 3.2$ billion for spinal fusion procedures in fiscal year 2011, making it the third largest CMS Centers for Medicare \& Medicaid Services expenditure behind total knee replacement and heart failure. It has also been the focus of attention in investigations to contain hospital costs. The increase in surgical procedures for the management of chronic pain and consequent escalation of healthcare costs has prompted the attention of policy makers as well.
\end{abstract}

Keywords: Back pain; Spinal fusion; Disability; Physicians

\section{Introduction}

The cost of spinal fusion surgeries has increased rapidly over time due to advances in bone-morphogenetic proteins (BMPs) that promote bone creation and remodeling and prolonged postoperative care needed in hospitals $[1,2]$. In spite of the focus of attention by different stakeholders, there are few examples of examination of surgical costs due to regional or other differences [3-5]. A previous study found that hospital mortality, length of stay, and costs were significantly less at the high-volume regional medical centers when compared with all other hospitals [6]. However, there is a general dearth of literature on the impact of non-clinical factors such as hospital location and types of payer on the costs of spinal fusion surgeries. This study examines the regional and payer type variations of hospital costs for spinal fusion procedures.

It has long been known that admission rates vary in different market areas partly because of physician practice styles [7]. Other studies have attempted to investigate the national trends in spinal fusion procedures performed. Frequency, utilization, and hospital charges of spinal fusion have increased at a higher rate than other notable inpatient procedures, as seen in a study of data from 1998 to 2008 [8]. We consider whether the costs of the spinal fusion procedures are uniform across different regions within the US. In addition to the regions, we examine the effects of payer types on differences in costs that might be evident.

\section{Theoretical Foundations}

The most directly applicable theory of price determination is the theory of supply and demand from microeconomics and it aims to explain the process of price determination. Although this theory has been to known to exist for a long time, its modern version is usually attributed to Marshall [9]. The model is commonly applied to wages in labor market but it has a wide applicability in a variety of markets ranging from information systems graduates [10] to interest rate determination due to money supply [11]. Drawing from its wide applicability, our model includes supply variables such as physicians available to perform spinal fusion and the number of procedures in a given area. Hospital charges are a direct measure of prices charged by hospitals for specific treatments.

\section{Literature Review}

This study employs a secondary analysis of the Healthcare Cost and Utilization Project's (HCUP) National Inpatient Sample (NIS) dataset to retrospectively examine whether there are regional and payer type differences of the costs of spinal fusion procedures. We examined the NIS data for years 2009, 2010, 2011 and 2012 - using a two-way analysis of variance. Beginning in 2012, the NIS was redesigned. It was formerly a sample of hospitals, and all discharges from those hospitals were retained. The new NIS starting with 2012 data is a sample of discharges from all hospitals participating in Healthcare Cost and Utilization Project's HCUP. Spinal fusion was classified as Diagnosis Related Groups (DRGs) 453 through 460 or Diagnosis Related Groups (DRGs) 471, 472 or 473 [6]. Based on this subset, there were 84,320 observations for 2009, 91,955 observations for 2010, 95,338 observations for 2011, and 87,920 observations for 2012. We used the TOTCHG column which contains the edited total charges as the total cost for the procedure. If the source provided the total charges with professional fees, then the professional fees were removed from the charge during Healthcare Cost and Utilization Project's (HCUP) processing to obtain the edited version of the charges [12,13]. In a small number of Healthcare Cost and Utilization Project's (HCUP) databases, professional fees were not removed from total charges because the data source could not provide the information. The Hospital region was obtained from the HOSP_REGION column which was gathered from the American Hospital Association (AHA) Annual Survey of Hospitals. Census region is defined by the U.S. Census Bureau. HOSP_REGION is used as a stratifier for both the Nationwide Inpatient Sample (NIS) and Nationwide Emergency Department Sample (NEDS). This is an important stratifier because practice patterns have been shown to vary substantially by region. The four regions were Northeast, Midwest, South and West which were coded with values 1 through 4 respectively. The payer was coded with values 1 through 6 representing Medicare, Medicaid, Private Insurance, Self-pay, No charge, and other. Our intent

*Corresponding author: Ravi Chinta, Department of Management, Huizenga College of Business and Entrepreneurship, USA, Tel: + 513-262-5512; E-mail: rchinta@nova.edu

Received February 06, 2019; Accepted February 14, 2019; Published February 21, 2019

Citation: Chinta R (2019) Variations of Hospital Costs of Spinal Fusion Procedures in Different Regions and Payer Types. J Spine 8: 433. doi 10.4172/2165-7939.1000433

Copyright: (c) 2019 Chinta R. This is an open-access article distributed under the terms of the Creative Commons Attribution License, which permits unrestricted use, distribution, and reproduction in any medium, provided the original author and source are credited. 
is to explore the regional and payer type differences in costs and also identify any temporal trends in the costs. Our model included both the main and interaction effects of hospital region and payer type [14,15]. We also do a post hoc analysis (in all cases where the main effects of both the payer type and hospital region are significant applying the Tukey HSD Honestly Significant Difference to the fitted model in each case to check if there are any pairwise comparative differences.

\section{Results}

The mean costs for all four years grouped by regions and payer types are reported in Tables 1 and 2 respectively and also diagrammatically represented in Figures 1 and 2. Both the tabulated data and diagrams convey a notable variation in charges due to differences in regions and payer types. A statistical analysis of the data confirms the inference that there are statistically significant differences for the years being studied.

For the year 2009, two-factor analysis of variance showed both the main effects of regions and payer types to be significant - Payer type $\mathrm{F}(5,83263)=111.856, \mathrm{p}<0.001$, and the hospital region $\mathrm{F}(3$, $83263)=1554.931, p<0.001$. The interaction effect between the payer type and hospital region was also found to be significant with $\mathrm{F}$ (15, $83263)=6.99, \mathrm{p}<0.001$ (Table 3).

Post hoc pairwise comparisons were made for both the main effects of regions and payer types with Tukey HSD (Honestly Significant Difference). All regional pairwise comparisons were statistically significant except for the South-Midwest. That is, only "South" and "Midwest" regions were similar in total charges and did not show any statistically significant differences. Similar pairwise statistically significant differences were revealed across all pairs of payer types except "Medicare" and "Medicaid" pair. These tabled results are available upon request from the author and not included here to save space.

For the year 2010, analysis of variance revealed both the main effects of regions and payer types to be significant - Payer type
$\mathrm{F}(5,91022)=45.70, \mathrm{p}<0.001$, and the hospital region with $\mathrm{F}(3$, $91022)=1129.67, \mathrm{p}<0.001$. The interaction effect between the payer type and hospital region was also found to be significant $\mathrm{F}(15,91022)=13.55$, $\mathrm{p}<0.001$ (Table 4).

Post hoc pairwise comparisons were made for both the main effects of regions and payer types with Tukey HSD (Honestly Significant Difference). All regional pairwise comparisons were statistically significant except for the Northeast-Midwest. That is, only "Northeast" and "Midwest" regions were similar in total charges and did not show any statistically significant differences. Similar pairwise statistically

\begin{tabular}{|c|c|c|c|c|}
\hline Year & Northeast & Midwest & South & West \\
\hline 2009 & $\$ 69,041$ & $\$ 77,967$ & $\$ 79,128$ & $\$ 116,736$ \\
\hline 2010 & $\$ 78,420$ & $\$ 79,288$ & $\$ 88,585$ & $\$ 116,455$ \\
\hline 2011 & $\$ 94,116$ & $\$ 78,167$ & $\$ 93,415$ & $\$ 135,707$ \\
\hline 2012 & $\$ 89,080$ & $\$ 84,257$ & $\$ 94,957$ & $\$ 131,590$ \\
\hline
\end{tabular}

Table 1: Mean charges aZfor different regions.

\begin{tabular}{|c|c|c|c|c|c|c|}
\hline Year & Medicare & Medicaid & $\begin{array}{c}\text { Private } \\
\text { insurance }\end{array}$ & Self-pay & No charge & Other \\
\hline 2009 & $\$ 90,954$ & $\$ 93,550$ & $\$ 79,694$ & $\$ 73,984$ & $\$ 82,674$ & $\$ 84,866$ \\
\hline 2010 & $\$ 94,434$ & $\$ 93,837$ & $\$ 87,026$ & $\$ 92,853$ & $\$ 105,791$ & $\$ 89,504$ \\
\hline 2011 & $\$ 104,925$ & $\$ 102,559$ & $\$ 92,168$ & $\$ 99,899$ & $\$ 104,427$ & $\$ 100,135$ \\
\hline 2012 & $\$ 103,958$ & $\$ 103,958$ & $\$ 93,933$ & $\$ 106,132$ & $\$ 94,472$ & $\$ 98,611$ \\
\hline
\end{tabular}

Table 2: Mean charges for different payer types.

\begin{tabular}{|c|c|c|c|c|c|}
\hline Variables & Df & Sum sq & Mean sq & F & p-value \\
\hline Payor & 5 & $2.58 \mathrm{E}+12$ & $5.15 \mathrm{E}+11$ & 111.856 & $0.001^{\text {***}}$ \\
\hline hosp_region & 3 & $2.15 \mathrm{E}+13$ & $7.16 \mathrm{E}+12$ & 1554.931 & $0.001^{\text {** }}$ \\
\hline Payor *hosp_region & 15 & $4.83 \mathrm{E}+11$ & $3.22 \mathrm{E}+10$ & 6.999 & $0.001^{\text {*** }}$ \\
\hline Residuals & 83263 & $3.83 \mathrm{E}+14$ & $4.60 \mathrm{E}+09$ & --- & --- \\
\hline
\end{tabular}

Table 3: The Anova table for total charges (Year 2009).

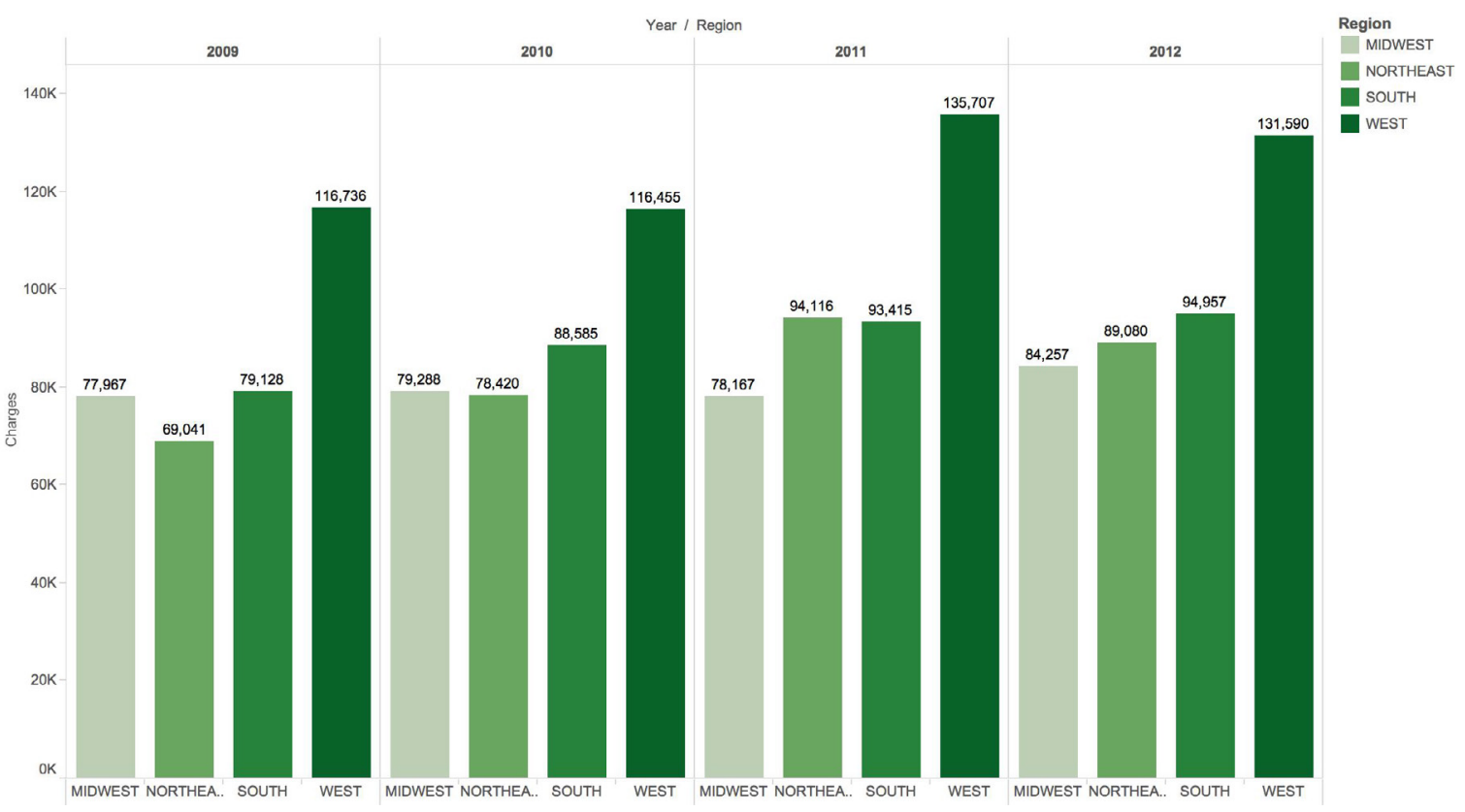

Figure 1: Mean spinal fusion costs in different regions for the years 2009-2012. 


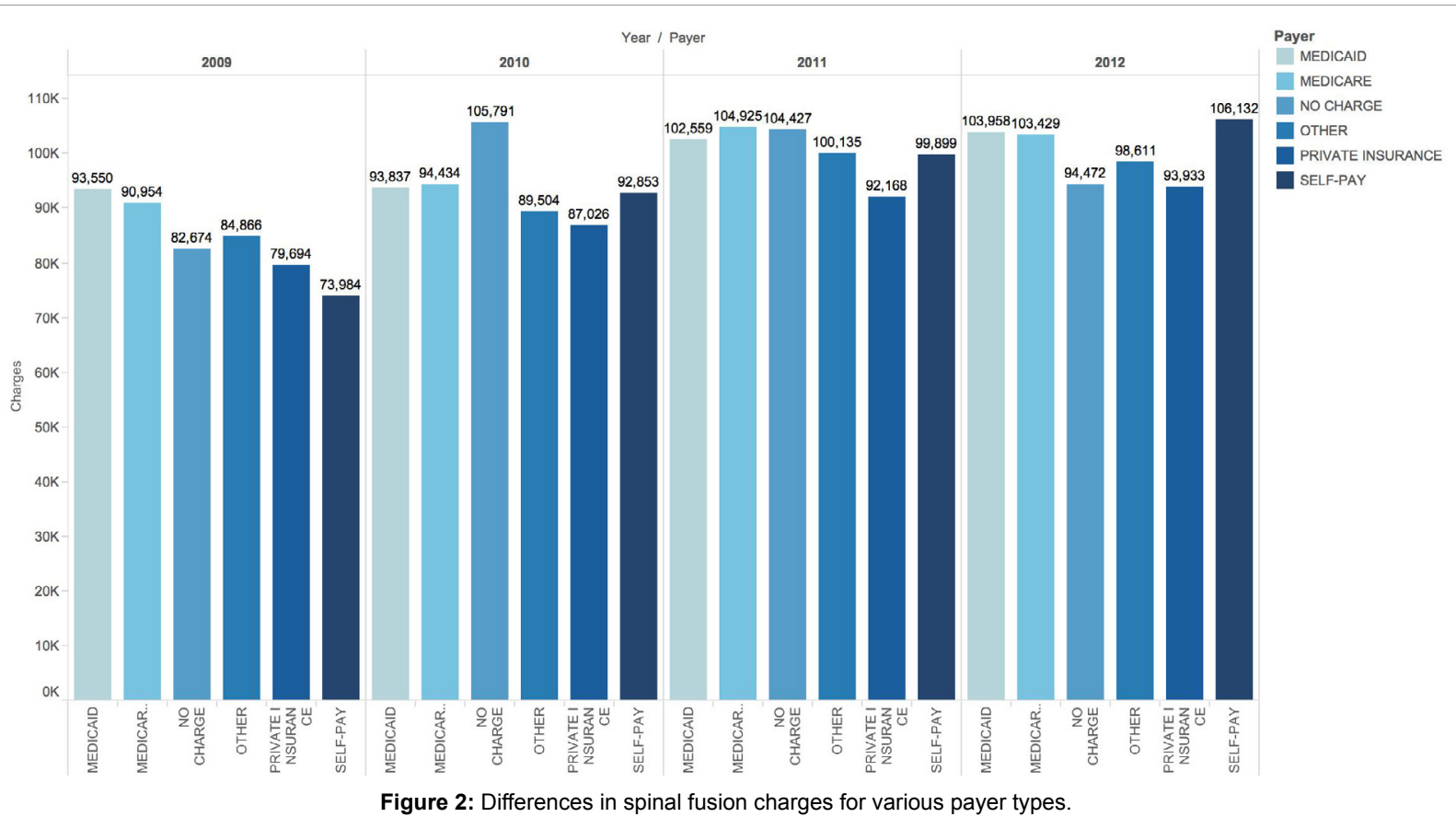

\begin{tabular}{|c|c|c|c|c|c|}
\hline Variables & Df & Sum sq & Mean sq & $\mathbf{F}$ & p-value \\
\hline Payor & 5 & $1.11 \mathrm{E}+12$ & $2.23 \mathrm{E}+11$ & 45.7 & $0.001^{\text {*** }}$ \\
\hline hosp_region & 3 & $1.65 \mathrm{E}+13$ & $5.50 \mathrm{E}+12$ & 1129.67 & $0.001^{\text {*** }}$ \\
\hline Payor *hosp_region & 14 & $9.23 \mathrm{E}+11$ & $6.60 \mathrm{E}+10$ & 13.55 & $0.001^{\text {*** }}$ \\
\hline Residuals & 91022 & $4.43 \mathrm{E}+14$ & $4.87 \mathrm{E}+09$ & --- & --- \\
\hline
\end{tabular}

Table 4: The Anova table for total charges (Year 2010).

\begin{tabular}{|c|c|c|c|c|c|}
\hline Variables & DF & Sum SQ & Mean SQ & F & p-value \\
\hline Payor & 5 & $3.24 \mathrm{E}+12$ & $6.48 \mathrm{E}+11$ & 104.6 & $0.001^{* * *}$ \\
\hline hosp_region & 3 & $3.57 \mathrm{E}+13$ & $1.19 \mathrm{E}+13$ & 1920.1 & $0.001^{* * *}$ \\
\hline Payor *hosp_region & 15 & $1.76 \mathrm{E}+12$ & $1.17 \mathrm{E}+11$ & 18.9 & $0.001^{* * *}$ \\
\hline Residuals & 93227 & $5.78 \mathrm{E}+14$ & $6.20 \mathrm{E}+09$ & --- & --- \\
\hline
\end{tabular}

Table 5: The Anova table for total charges (Year 2011).

\begin{tabular}{|c|c|c|c|c|c|}
\hline Variables & DF & Sum SQ & Mean SQ & F & p-value \\
\hline Payor & 5 & $1.776 \mathrm{e}+12$ & $3.552 \mathrm{e}+11$ & 54.312 & $0.001^{\text {***}}$ \\
\hline hosp_region & 3 & $2.374 \mathrm{e}+13$ & $7.913 \mathrm{e}+12$ & 1209.832 & $0.001^{\text {***}}$ \\
\hline Payor *hosp_region & 15 & $9.495 \mathrm{e}+11$ & $6.330 \mathrm{e}+10$ & 9.678 & $0.001^{\text {***}}$ \\
\hline Residuals & 83809 & $5.482 \mathrm{e}+14$ & $6.541 \mathrm{e}+09$ & & \\
\hline
\end{tabular}

Table 6: The Anova table for total charges (Year 2012).

significant differences were revealed across all pairs of payer types except "Medicare" and "Medicaid" pair. These tabled results are available upon request from the author and not included here to save space.

For the year 2011, both the main effects of regions and payer types are significant - Payer type $F(5,93227)=104.6, p<0.001$, and the hospital region $\mathrm{F}(3,93227)=1920.1, \mathrm{p}<0.001$. The interaction effect between the payer type and hospital region was also found to be significant $\mathrm{F}(15,93227)=18.9, \mathrm{p}<0.001$ (Table 5).

Post hoc pairwise comparisons were made for both the main effects of regions and payer types with Tukey HSD (Honestly Significant Difference). All regional pairwise comparisons were statistically significant except for the South-Northeast. That is, only "South" and
"Northeast" regions were similar in total charges and did not show any statistically significant differences. Similar pairwise statistically significant differences were revealed across all pairs of payer types except "Medicare" and "Medicaid" pair. These tabled results are available upon request from the author and not included here to save space.

For the year 2012, both the main effects of regions and payer types to be significant - Payer type F $(5,83809)=54.312, \mathrm{p}<0.001$, and the hospital region $\mathrm{F}(3,83809)=1209.832, \mathrm{p}<0.001$. The interaction effect between the payer type and hospital region was also found to be significant $\mathrm{F}(15,83809)=9.678, \mathrm{p}<0.001$. (Table 6).

Post hoc pairwise comparisons were made for both the main effects of regions and payer types with Tukey HSD (Honestly Significant Difference). Interestingly, all regional pairwise comparisons were statistically significant. That is, all regions showed statistically significant differences. Similar pairwise statistically significant differences were revealed across all pairs of payer types except "Medicare" and "Medicaid" pair. These tabled results are available upon request from the author and not included here to save space.

\section{Discussion}

At an aggregate level, spinal fusion is a high-frequency procedure done in US hospitals and contributed to substantial healthcare costs incurred in hospitals in the US. Our study reveals that the total charges for spinal fusion vary widely by payer type and hospital region. The variation of total charges for spinal fusion is statistically significant. Several implications arise from these empirical findings which are detailed next.

The wide variation of total charges by payer type found in our study does not tell us much about the actual costs incurred for spinal fusion procedures which depend on covariates such as patient diversity, complications and comorbidities. Total charge for spinal fusion is often determined by the contractual agreement between the provider and the payer based on an average estimation of costs for reimbursement for a particular sub-aggregate of spinal fusion procedures. Thus, a significant 
cross-subsidization of costs occurs in the use of total charges by payer type. The implication of this finding for healthcare cost accounting becomes significant. In the quest for evidence-based medicine the gap between total charge and actual costs incurred must be narrowed.

The variation of total charges by hospital region leads to an identification of high-cost and low-cost regions for spinal fusion procedures across US. Such empirical identification of high and low cost hospitals provides a knowledge landscape that can define potential collaborative relationships between high-cost and low-cost hospitals. Perhaps, directing patient flows from high-cost to low-cost hospitals could become an IT-based cost minimization protocol where it is physically feasible.

The interaction between payer type and hospital region is an interesting finding that calls for more future research to determine of the interaction effects are positive or negative and thereby leading to some systems redesign that allow reinforcing effects that tend to lower total charges. Specifically, Medicare payer in South region may be more effective in its interaction effects on total charges when compared to private payers in North East. Such specific empirical interaction effects can inform systems redesign in effective management of total charges for spinal fusions in hospitals.

\section{Conclusion}

Our study contributes to the ongoing unraveling of empirically based relationships amongst variables that impact total charges for spinal fusion procedures in hospitals over 2009-2012 period. Our study must be considered a part of ongoing research that helps in understanding the complex healthcare cost management picture. Future research based on our findings will help uncover a more granular picture that can help in systems redesign aimed at more effective management of total charges for spinal fusion procedures in hospitals.

\section{References}

1. Cahill KS, Chi JH, Day A, Claus EB (2009) Prevalence, complications, and hospital charges associated with use of bone-morphogenetic proteins in spinal fusion procedures. JAMA 302: 58-66.
2. Lee AF, Sirkka LJ, Bradley CW (2000) The supply and demand of information systems doctorates: Past, present, and future. MIS Quarterly 24: 355-380.

3. Gordon TA, Burleyson GP, Tielsch JM, Cameron JL (1995) The effects of regionalization on cost and outcome for one general high-risk surgical procedure. Ann Surg 221: 43-49.

4. Herman B (2014) Top 20 costliest surgical procedures performed in hospitals. Hospital CFO Report, USA.

5. Little KM, Jevsevar DS, Shea KG, Wright KK, Simon AW, et al. (2014) The Incremental Hospital Cost of Complications among Medicare Beneficiaries Undergoing Spinal Fusion. The Spine Journal 14: S157.

6. Lo KW, Jiang T, Gagnon KA, Nelson C, Laurencin CT (2014) Small-molecule based musculoskeletal regenerative engineering. Trends in biotechnol 32: 7481.

7. Manchikanti L, Helm S, Pampati V, Racz GB (2014) Cost utility analysis of percutaneous adhesiolysis in managing pain of post-lumbar surgery syndrome and lumbar central spinal stenosis. Pain Pract 15: 414-422.

8. Marshall A (2009) Principles of economics: Unabridged eighth edition. London, Macmillan and Co.

9. Martin BI, Deyo RA, Mirza SK, Turner JA, Comstock BA, et al. (2008) Expenditures and health status among adults with back and neck problems. JAMA 299: 656-664

10. Miller DC, Gust C, Dimick JB, Birkmeyer N, Skinner J, et al. (2011) Large variations in Medicare payments for surgery highlight savings potential from bundled payment programs. Health Aff 30: 2107-2115.

11. Rajaee SS, Bae HW, Kanim LE, Delamarter RB (2012) Spinal fusion in the United States: An analysis of trends from 1998 to 2008. Spine 37: 67-76.

12. Gregory FU, Lawrence SR, William LS (2000) Principles of money, banking, and financial markets (10th edn) pp: 431-438, 465-476.

13. Report on Medicare Compliance (2012) Spinal fusion is new PEPPER target, with focus on medical necessity of procedure 21: 5 .

14. Terhune $C$ (2015) Medicare plans big payment changes for knee and hip replacements. Los Angeles Times, USA.

15. Wennberg JE, McPherson K, Caper $\mathrm{P}$ (1984) Will payment based on diagnosisrelated groups control hospital costs? N Engl J Med 311: 295-300. 